\title{
Extinction in the Star Cluster SAI 113 and Galactic Structure in Carina
}

\author{
Giovanni Carraro ${ }^{1}$, David G. Turner ${ }^{2}$, Daniel J. Majaess ${ }^{2}$, Gustavo L. Baume ${ }^{3}$, Roberto Gamen ${ }^{3}$, and José A. Molina Lera ${ }^{3}$ \\ ${ }^{1}$ Dipartimento di Fisica e Astronomia, Universitá di Padova, Vicolo Osservatorio 3, I-35122, Padova, Italy; giovanni.carraro@unipd.it \\ ${ }^{2}$ Department of Astronomy and Physics, Saint Mary’s University, Halifax, NS B3H 3C3, Canada; turner@ap.smu.ca, dmajaess@ns.sympatico.ca \\ ${ }^{3}$ Facultad de Ciencias Astronómicas y Geofísicas (UNLP), IALP (CONICET, UNLP), Paseo del Bosque s/n, \\ La Plata, Argentina; gbaume@fcaglp.unlp.edu.ar, rgamen@fcaglp.unlp.edu.ar, jalejom1@ fcaglp.unlp.edu.ar \\ Received 2016 October 18; revised 2017 January 18; accepted 2017 January 23; published 2017 March 10
}

\begin{abstract}
Photometric CCD $U B V I_{C}$ photometry obtained for 4860 stars surrounding the embedded southern cluster SAI 113 (Skiff 8) is used to examine the reddening in the field and derive the distance to the cluster and nearby van Genderen 1. Spectroscopic color excesses for bright cluster stars, photometric reddenings for A3 dwarfs, and dereddening of cluster stars imply that the reddening and extinction laws match results derived for other young clusters in Carina: $E_{U-B} / E_{B-V} \simeq 0.64$ and $R_{V} \simeq 4$. SAI 113 displays features that may be linked to a history of dynamical interactions among member stars: possible circumstellar reddening and rapid rotation of late B-type members, ringlike features in star density, and a compact core, with most stars distributed randomly across the field. The group van Genderen 1 resembles a stellar asterism, with potential members distributed randomly across the field. Distances of $3.90 \pm 0.19 \mathrm{kpc}$ and $2.49 \pm 0.09 \mathrm{kpc}$ are derived for SAI 113 and van Genderen 1, respectively, with variable reddenings $E_{B-V}$ ranging from 0.84 to 1.29 and 0.23 to 1.28 . The SRC variables CK Car and EV Car may be outlying members of van Genderen 1, thereby of use for calibrating the periodluminosity relation for pulsating $\mathrm{M}$ supergiants. More importantly, the anomalous reddening and extinction evident in Carina and nearby regions of the Galactic plane in the fourth quadrant impact the mapping of spiral structure from young open clusters. The distribution of spiral arms in the fourth quadrant may be significantly different from how it is often portrayed.
\end{abstract}

Key words: dust, extinction - Galaxy: structure - Hertzsprung-Russell and C-M diagrams open clusters and associations: individual (SAI 113, van Genderen 1) - stars: distances

\section{Introduction}

Researchers at the Sternberg Astronomical Institute (SAI) in Moscow recently compiled initial parameters for a sample of about 170 newly detected and previously unstudied open star clusters (Glushkova et al. 2010) discovered from analyses of 2MASS $J H K_{s}$ photometry (Cutri et al. 2003) for Galactic fields covered by the 2MASS survey (Skrutskie et al. 2006), many subjected to additional study using $U B V I$ data. Object 113 in the sample, SAI 113, at $\left(\alpha_{2000}, \delta_{2000}\right)=(10: 22: 43.6,-59: 30: 20)$, is one of 23 such groups, separated into a table of "embedded clusters," for which parameters are not given, typically because of large scatter in the 2MASS colors. SAI 113 is the primary subject of the present study.

The cluster was noticed previously by Brian Skiff while deriving coordinates for stars near the luminous blue variable HR Carinae observed by van Genderen et al. (1991). The brightest stars in SAI 113 are numbered 78, 82, 83, 84, and 85 on an inset of van Genderen's Figure 1, which is reversed from the field's appearance in the sky. The group is therefore also designated as Skiff 8 in the most recent version of Bruno Alessi's open cluster catalog (B. Alessi 2016, private communication).

A separate, sparse group of early-type stars is visible a few arcminutes east of HR Car on the van Genderen et al. (1991) chart at $\left(\alpha_{2000}, \delta_{2000}\right)=(10: 22: 29,-59: 38: 30)$, and is designated as van Genderen 1 (B. Alessi 2016, private communication). SAI 113 and van Genderen 1 are both fairly compact, and members of both display differential reddening according to the transformed Walraven photometry for the stars tabulated by van Genderen et al. (1991), as well as by the dispersion in their 2MASS colors. The present study was initiated in order to fill the void in our knowledge of both groups, with an initial focus on SAI 113, particularly given the location of the two groups in the rich complex of young stars, open clusters, and associations belonging to and surrounding the Eta Carinae complex.

A blue light image centered roughly on SAI 113 is presented in Figure 1, where it can be noted that the term "embedded" refers to the appearance on colorized images of the 2MASS survey (Skrutskie et al. 2006), displayed as a BW inset to Figure 1, of the small clump of faint stars at its core, in the immediate lower right of the image. van Genderen 1 lies above the lower right margin of Figure 1. Except for the latter, bright stars display no obvious concentration toward either group. Rather, they are more or less evenly distributed across the field, possibly concentrating toward the margins of Figure 1, akin to the ringlike distribution in the cluster Collinder 70, the ellipsoidal group of stars surrounding $\epsilon$ Orionis and referred to as the Orion Stellar Ring (see Schild \& Cowley 1971), as well as to the ringlike concentration of stars surrounding the anonymous cluster near HD 18391 (Turner et al. 2009). Both seem to be in advanced stages of dissolution into the surrounding field, which may also be true of SAI 113 and van Genderen 1.

Star counts were made for SAI 113 to the limits of the 2MASS survey, as illustrated in Figure 2. Stars were counted in rings about the cluster center of Glushkova et al. (2010), following the statistical approach of van den Bergh \& Sher (1960). One arcminute rings were adopted, except for an additional half arcminute ring at the cluster center. According to the counts, the cluster has a nuclear radius of $r_{n}=1.5$ and a coronal radius of $R_{c}=6^{\prime}$ in the notation of Kholopov (1969), 


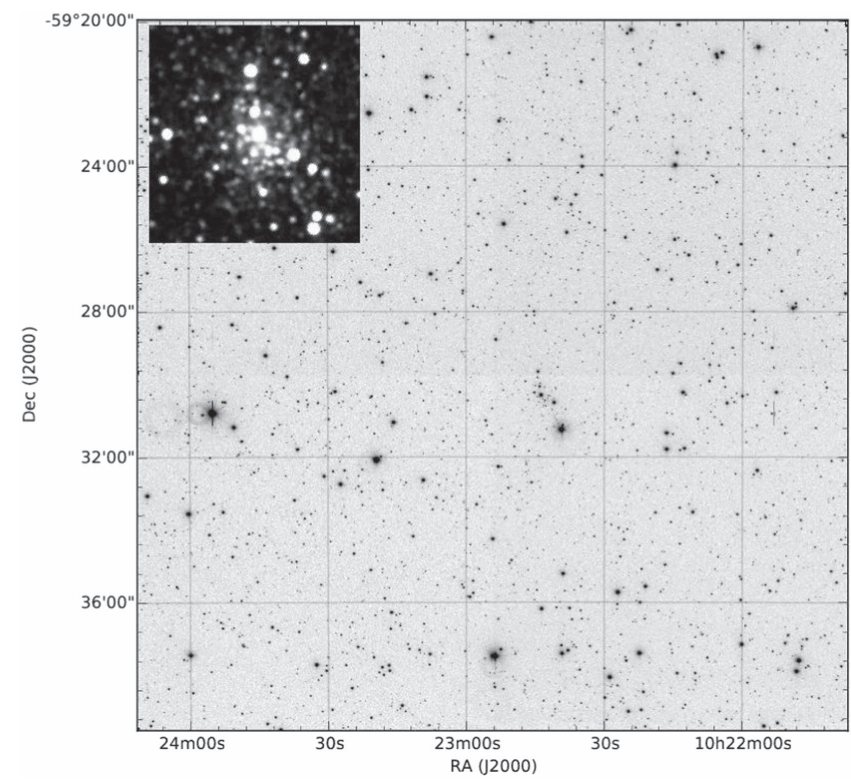

Figure 1. The $20^{\prime} \times 20^{\prime}$ field of SAI 113 centered on $\left(\alpha_{2000}\right.$ $\left.\delta_{2000}\right)=(10: 22: 54,-59: 30: 00)$, as viewed in blue light, with north up and east to the left. The clump of stars at the cluster core, shown separately in the inset according to its appearance in 2MASS colorized images, lies immediately southwest of the field center, while van Genderen 1 lies just above the lower right margin of the field.

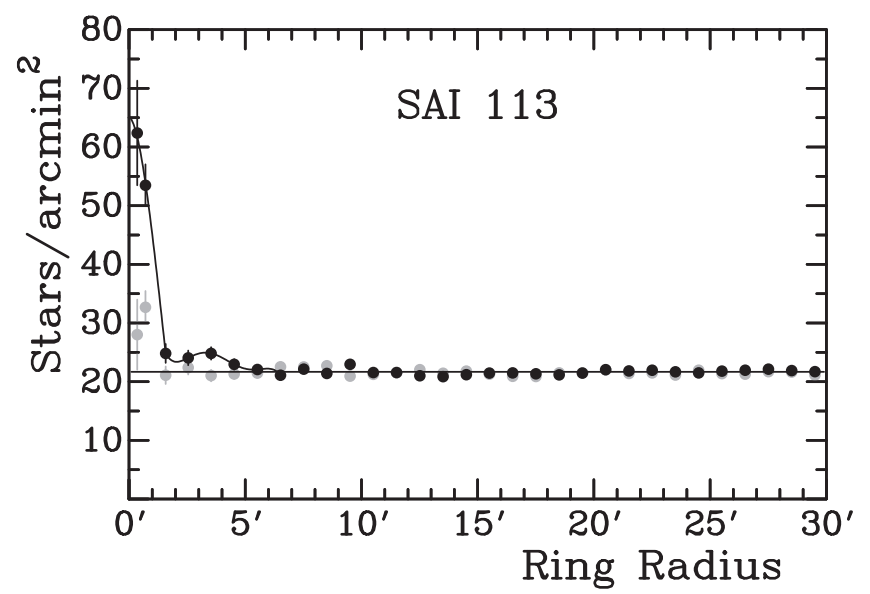

Figure 2. Star counts off the 2MASS survey to 30 arcmin from the centers for SAI 113 (black points) and van Genderen 1 (gray points), with uncertainties tied to Poisson statistics. The field level was established from counts in the 15-30 arcmin rings. The curve is a schematic illustrating likely variations in star density for the inner regions of SAI 113.

with $244 \pm 51$ cluster members lying above the field level of $21.68 \pm 0.05$ stars $\operatorname{arcmin}^{-2}$ within $6^{\prime}$ of the cluster center. The subtle blips in the counts around $3^{\prime}$ and $9^{\prime}$ from the cluster center may be real, since they lie several $\sigma$ above the field star level. They suggest the existence of inner ringlike structures such as those in the outer regions of Collinder 70 and Anon (HD 18391), other dissolving clusters. Otherwise, outlying stars tend to be distributed randomly across the field, more like an association than an open cluster.

Similar star counts were made for van Genderen 1 using the cluster center cited previously, with results plotted in Figure 2 as gray points. van Genderen 1 is clearly a less prominent group than SAI 113, and may represent merely the dissolved remains of a former young cluster. This is also the impression obtained from casual examination of the field on Sky Survey

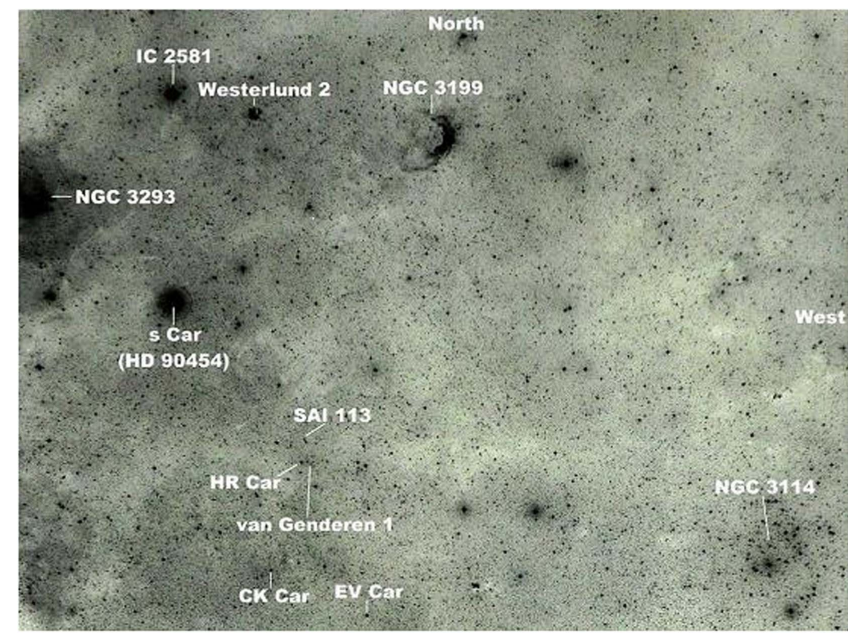

Figure 3. The roughly $3.5 \times 5^{\circ}$ field west of the Eta Carinae complex containing SAI 113 and other objects discussed in the text. Note the numerous opaque dust clouds running across the field that seem to merge near SAI 113. The image is from a $J$-band (blue) exposure from the ESO-SRC survey.

images. The counts imply the existence of just $4 \pm 17$ group members above field level within $2^{\prime}$ of the adopted center.

The location of SAI 113 and van Genderen 1 in the Carina complex is of particular interest because of the possibility of using them to test the extent of the "anomalous" extinction that has been documented for most of the clusters and stellar groups in the region (Turner 2012a; Carraro et al. 2013). According to Neckel \& Klare (1980), the extinction for this direction of the Galaxy arises in dust clouds roughly $1 \mathrm{kpc}$ distant, while deep images of the field in blue light (Figure 3) reveal that strands of the same dust cloud permeate all of the "anomalous" regions of Carina, including those that converge near SAI 113. Prominent dust strands in Figure 3 can be traced northward to Westerlund 2 and IC 2581, and eastward to NGC 3293 and (outside the image) to the clusters of the Great Carina Nebula: Trumpler 14, Trumpler 15, Trumpler 16, Collinder 228, Collinder 236, Ruprecht 93, and Anon (WR 38/38a; Turner 2012a). The anomalies relate to a shallow reddening slope of $E_{U-B} / E_{B-V} \simeq 0.64$ on the Johnson system and a large ratio of total-to-selective extinction of $R_{V}=A_{V} / E_{B-V} \simeq 4$. The anomaly for $R_{V}$ is particularly important since it affects the derived distances to clusters in Carina: $1.9-2.3 \mathrm{kpc}$ relative to original estimates of 2.5-3.4 kpc (see Turner 2012a). Such a change dramatically affects how we picture the width and orientation of the Carina-Sagittarius arm of the Galaxy.

\section{Observations and Data Reduction}

$U B V I_{C}$ images centered on SAI 113 were obtained on two nights (17 and 22) of 2009 March at Cerro Tololo InterAmerican Observatory (CTIO) using the Y4KCAM camera on the $1.0 \mathrm{~m}$ telescope operated by the SMARTS consortium, as noted in Table 1. The camera was equipped with an STA $4064 \times 4064$ CCD with $15 \mu$ pixels, a setup that provided direct imaging over a field of view (FOV) of 20 . $0 \times 20$ ' 0 at a scale of 0 .' 289 pixels $^{-1}$. The typical fullwidth half-maximum for the data was about 0.9 . Figure 1 is based on a $60 \mathrm{~s}$ exposure of the field in the $B$ band.

The CTIO images were processed with the IRAF packages CCDRED and DAOPHOT using the point-spread function (PSF) method of Stetson (1987) to extract instrumental 
Table 1

UB VI Photometric Imaging of SA 113 in 2009 March

\begin{tabular}{llll}
\hline \hline Date & Filter & Exposures (s) & Airmass X \\
\hline March 17 & $U$ & $30,200,2000$ & $1.17-1.18$ \\
& $B$ & $20,150,1500$ & $1.21-1.22$ \\
& $V$ & $10,100,900$ & $1.32-1.34$ \\
March 22 & $I$ & $10,100,900$ & $1.27-1.28$ \\
& $U$ & $30,200,2000$ & 1.16 \\
& $B$ & $20,150,1500$ & 1.19 \\
& $V$ & $10,100,900$ & $1.26-1.27$ \\
& $I$ & $10,100,900$ & $1.23-1.24$ \\
\hline
\end{tabular}

Table 2

Calibration Coefficients for 2009 March 22

\begin{tabular}{llll}
\hline \hline$u 1$ & $-0.851 \pm 0.006$ & $b 1$ & $-2.064 \pm 0.010$ \\
$u 2$ & 0.45 & $b 2$ & 0.25 \\
$u 3$ & $-0.029 \pm 0.009$ & $b 3$ & $0.128 \pm 0.009$ \\
$\mathrm{rms}$ & \pm 0.08 & $\mathrm{rms}$ & \pm 0.08 \\
$v 1_{b v}$ & $-2.129 \pm 0.008$ & $\mathrm{v} 1_{v i}$ & $-2.119 \pm 0.006$ \\
$v 2_{b v}$ & 0.16 & $v 2_{\mathrm{vi}}$ & 0.16 \\
$v 3_{b v}$ & $-0.021 \pm 0.008$ & $v 3_{v i}$ & $-0.032 \pm 0.005$ \\
$\mathrm{rms}$ & \pm 0.06 & $\mathrm{rms}$ & \pm 0.02 \\
$i 1$ & $-1.321 \pm 0.005$ & $\ldots$ & $\ldots$ \\
$i 2$ & 0.08 & $\ldots$ & $\ldots$ \\
$i 3$ & $-0.016 \pm 0.004$ & $\ldots$ & $\ldots$ \\
$\mathrm{rms}$ & \pm 0.04 & $\ldots$ & $\ldots$ \\
\hline
\end{tabular}

magnitudes. The PSF for each image was obtained for several isolated, spatially well-distributed, bright stars in the field $(\sim 20$ in the sample). The PSF photometry for individual objects was then aperture corrected for each filter, where the corrections were computed from aperture photometry on the same stars used as PSF models. All resulting tables were combined by the DAOMASTER code (Stetson 1992), where only those objects with $\chi^{2} \leqslant 3$ and abs(sharp) $\leqslant 0.5$ were included, in order to avoid spurious detections. The functions $\chi^{2}$ and abs(sharp) are standard diagnostics in DAOPHOT used in the automatic flux detection mode to measure the quality of a PSF fit relative to the adopted PSF model, and to monitor the brightness of detections and avoid defects caused by bad pixels. The night of 2009 March 22 was used as a photometric reference.

Our $U B V I_{C}$ data were calibrated through observations of about 70 standard stars in Landolt (1992) fields SA 98 and PG $1047+003$, the former providing very good color coverage. Aperture photometry was then carried out for all stars using the PHOTCAL package with transformation equations of the form:

$$
\begin{gathered}
u=U+u 1+u 2(U-B)+u 3 X \\
b=B+b 1+b 2(B-V)+b 3 X \\
v=V+v 1_{b v}+v 2_{b v}(B-V)+v 3_{b v} X \\
v=V+v 1_{v i}+v 2_{v i}(V-I)+v 3_{v i} X \\
i=I_{C}+i 1+i 2(V-I)+i 3 X,
\end{gathered}
$$

where $U B \quad V I_{C}$ and $u b v i$ are standard and instrumental magnitudes, respectively; $X$ is the airmass of the observation; and typical values for the extinction coefficients for CTIO were adopted (see Baume et al. 2011). Equation (3) was used to derive $V$ magnitudes when the $B$ magnitude was available; otherwise Equation (4) was used. The calibration coefficients for the night of 2009 March 22 are summarized in Table 2.

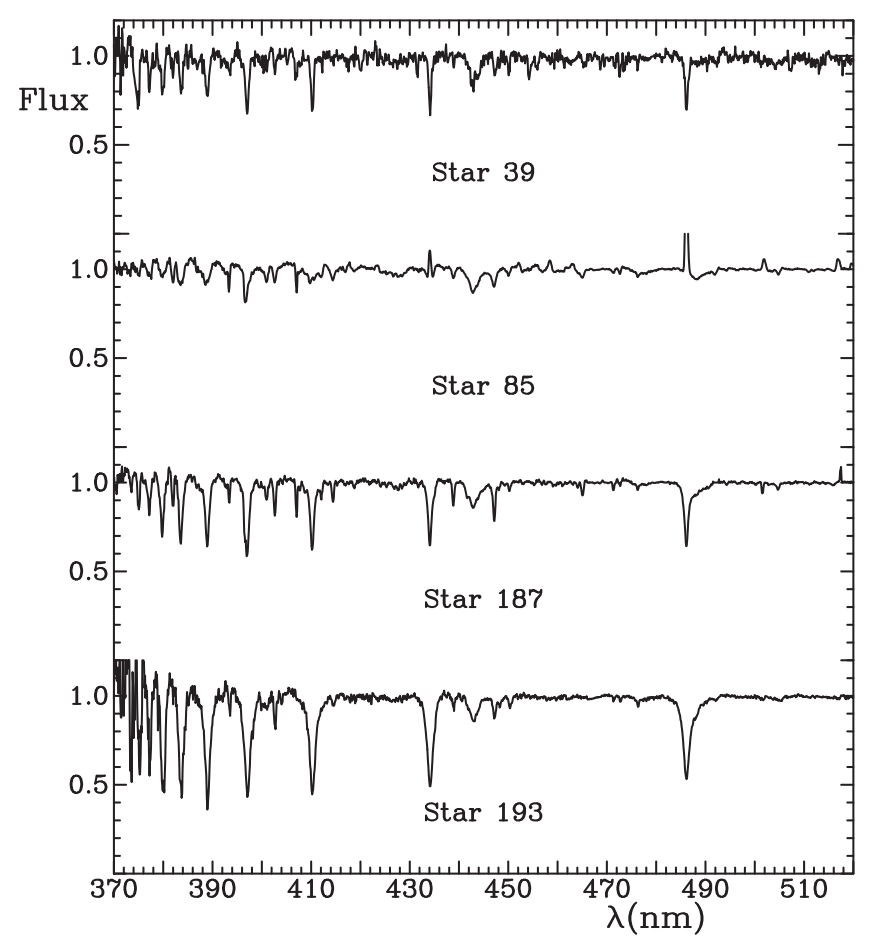

Figure 4. Normalized spectra for bright stars in SAI 113.

World Coordinate System header information was obtained for each image using the ALADIN tool and 2MASS data (Skrutskie et al. 2006). Baume et al. (2009) describe the procedure used to perform an astrometric calibration of the data. That yielded reliable astrometry with uncertainties in position of order $\sim 0$." 12 .

The STILTS tool was used to manipulate tables and to crosscorrelate the $U B V I_{C}$ and $2 \mathrm{MASS} J H K_{s}$ data. The result was a catalog with astrometric/photometric information on the detected objects covering an FOV of approximately $20^{\prime} \times 20^{\prime}$. The complete catalog will be made available in electronic form on the CDS website.

Spectra were also obtained for four of the bright stars in SAI 113 using the Gemini Multi-object Spectrograph in long-slit mode on the $8 \mathrm{~m}$ telescope of Gemini South, Chile. The observations were obtained in poor weather service observing periods of 2016 July, with a 0.75 arcsec slit width and grating B600, providing a typical resolving power of $\sim 1200$. The normalized spectra for the observed stars represent the average of four integrations and are presented in Figure 4, with spectral classifications and derived parameters summarized in Table 3. The spectra have been cleaned of a few obvious artefacts not originating in the stars, and also restricted to the blue-green spectral region normally used for classification on the MK system.

A fifth star was added to Table 3 (star 34), where the classification is from the third release of the Galactic O-Star Spectroscopic Survey, GOSSS (Maíz Apellániz et al. 2016). That survey also includes star 39, with the latter being classified there as $\mathrm{O} 7 \mathrm{~V}((\mathrm{f})) \mathrm{z}$, virtually identical to the present classification, the " $\mathrm{z}$ " denoting strong He II $\lambda 4686$ absorption. Star 85 was detected as an emission-line star previously by Henize (1976), and has the designation HEN 3-406. Reddenings for the hotter Be stars are affected by excess continuum emission according to Schild \& Romanishin (1976), 
Table 3

Spectroscopic Results for SAI 113 Stars

\begin{tabular}{lllllllll}
\hline \hline Star & $V$ & $B-V$ & $U-B$ & Sp.T. & $E_{B-V}$ & $E_{U-B}$ & $V-M_{V}$ \\
\hline $34^{\mathrm{a}}$ & 11.88 & 0.72 & -0.31 & O8 V(n) & 1.03 & 0.82 & 16.98 \\
$39^{\mathrm{b}}$ & 12.02 & 0.63 & -0.39 & O7 V(f))n & 0.95 & 0.77 & 16.82 \\
$85^{\mathrm{c}}$ & 12.82 & 0.83 & -0.40 & B1 Vnne & 0.95 & 0.62 & 12.87 \\
$187^{\mathrm{d}}$ & 13.88 & 0.74 & -0.23 & B1.5 Vn & 0.99 & 0.67 & 13.02 \\
$193^{\mathrm{e}}$ & 13.94 & 0.77 & -0.15 & B2.5 Vn & 0.99 & 0.64 & 17.33 \\
\hline
\end{tabular}

Notes.

a 2MASS J10224377-5930182.

b 2MASS J10224096-5930305.

c 2MASS J10224440-5929394, HEN 3-406.

d 2MASS J10223910-5931080.

e 2MASS J10224587-5932420.

amounting to systematic excesses of 0.15 in $E_{B-V}$ and -0.05 in $E_{U-B}$. The reddenings for star 85 in Table 3 have therefore been adjusted for that effect. The adopted absolute magnitudes used to estimate distance moduli have also been adjusted according to prior experience, indicating that dwarfs classified as nn, n, or (n), as well as dwarf Be stars, appear to have luminosities comparable to subgiants (class IV) rather than dwarfs (class V).

\section{Analysis of the Photometric Data}

The photometric $U B V$ data for stars lying within the boundaries of Figure 1 were tested by various means, with simple dereddening providing important results. It was found, for example, that dereddening solutions for stars with $V \geqslant 19$ were generally unrealistic when compared with those for brighter stars, so the analysis was restricted to objects with $V<19$.

The spectra for bright cluster stars listed in Table 3 have been classified on the MK system, here for 39, 85, 187, and 193, and by Maíz Apellániz et al. (2016) for 34, and according to the discussion of Section 1, generated the color excesses summarized in the table and plotted in Figure 5. The reddenings of the $\mathrm{B}$ stars are a close fit to an extinction law described by $E_{U-B}=0.64 E_{B-V}+0.02 E_{B-V}^{2}$, where the slope is that found previously for Carina clusters (Turner 2012a; Carraro et al. 2013) and the curvature term is that obtained for Galactic fields by Turner (1989). The exceptions to such a close fit are the two O-type stars, which are offset by +0.15 in $E_{U-B}$ from the above reddening line. It may be that both are affected by continuum emission, although it can be noted that both lie in heavily populated regions of SAI 113 and their photometry may simply be affected by crowding. It seems unlikely that the reddening law for $\mathrm{O}$ stars in the region differs from that for $\mathrm{B}$ stars. All five stars lie within $2^{\prime}$ of the center of SAI 113 and share common reddenings averaging $E_{B-V}=0.98 \pm 0.03 \sigma$ and common intrinsic distance moduli averaging $V_{0}-M_{V}=$ $13.03 \pm 0.25$, for $R_{V}=4$.

Color-color diagrams also provide information about reddening laws, since reddened objects corresponding intrinsically to the hottest O-type stars, or to stars lying near the A3 V "kink" in the intrinsic color-color relation, provide direct information about the reddening line's likely slope $E_{U-B} / E_{B-V}$, which in turn appears to be related to the ratio of total-to-selective extinction $R_{V}=A_{V} / E_{B-V} \quad$ (Turner et al. 2014).
The $U B V$ data for stars lying within $6^{\prime}$ of the center of SAI 113 are analyzed in Figure 6, which plots color-color and color-magnitude diagrams from the CCD observations. It is evident from both diagrams that the cluster field appears to exhibit a small amount of differential reddening and extinction, with a minimum reddening of $E_{B-V} \simeq 0.88$ applying to reddened cluster B-type stars. Foreground and line-of-sight stars appear to exhibit reddenings of $E_{B-V} \leqslant 0.20$, for the most part. In addition, the reddening for the main clump of B-type stars ranges from the minimum noted previously to values apparently as large as $E_{B-V} \simeq 1.28$, although only late B-type stars exhibit reddenings that large; most are less reddened than $E_{B-V} \simeq 1.08$. The larger reddenings all apply to mid- to late Btype stars, which typically exhibit large rotational velocities. They may exhibit an apparent excess reddening arising from rotational effects on the stellar continua, or possibly to a component of circumstellar reddening similar to that noted for late B-type stars in the open cluster Roslund 3 (Turner 1993, 1996).

Note the clump of stars in the upper section of Figure 6 that appear to be reddened from the A3 kink, matching a reddening law of slope $E_{U-B} / E_{B-V}=0.64$, as found spectroscopically. An identical feature in the color-color diagrams for other stars in the field of Figure 1 (see Figures 8 and 9) argues that the reddening law in the region matches that found in nearby Carina fields.

The zero-age main-sequence (ZAMS) relation tabulated by Turner $(1976 a, 1979)$ is plotted in the lower portion of Figure 6 for a reddening of $E_{B-V}=0.10$ and intrinsic distance modulus of $V_{0}-M_{V}=11.83$ (see later) in order to illustrate how lessreddened foreground stars are distributed in the colormagnitude diagram of SAI 113. Even with such stars removed from consideration, it is difficult to make a ZAMS fit for the B-type members of SAI 113 from the Figure 6 data by themselves, because of the deleterious effects of differential reddening and extinction. The plotted ZAMS fits for those stars are for $R_{V}=4$ and $V_{0}-M_{V}=12.95$ at reddenings of $E_{B-V}=0.88$ and 1.28 .

The implied presence of differential interstellar reddening in the field of SAI 113 from Figure 3 dictates that a detailed analysis of the photometric data requires the use of the variable-extinction method (see, for example, Turner 1976a, 1976b). By such a procedure, the data for reddened early-type stars throughout the field of Figure 1, not just in SAI 113, were dereddened to the intrinsic zero-age zero-rotation main sequence (ZAZRMS; see Turner 2016) using $U B V$ reddening lines of slope $E_{U-B} / E_{B-V}=0.64$. It was then possible to infer 


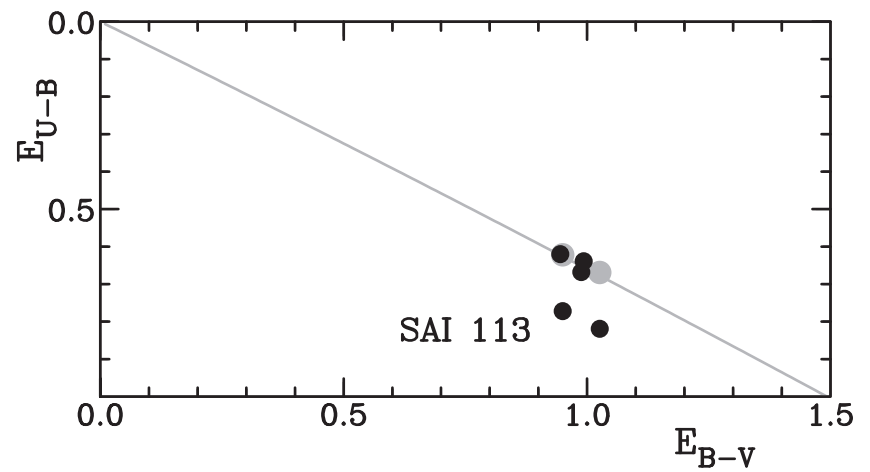

Figure 5. $U B V$ color excesses for spectroscopically observed stars in SAI 113 plotted with respect to a reddening line (in gray) described by $E_{U-B}=0.64$ $E_{B-V}+0.02 E_{B-V}^{2}$. Alternate $E_{U-B}$ reddenings for the $\mathrm{O}$ stars adjusted by -0.15 are plotted as larger gray points.

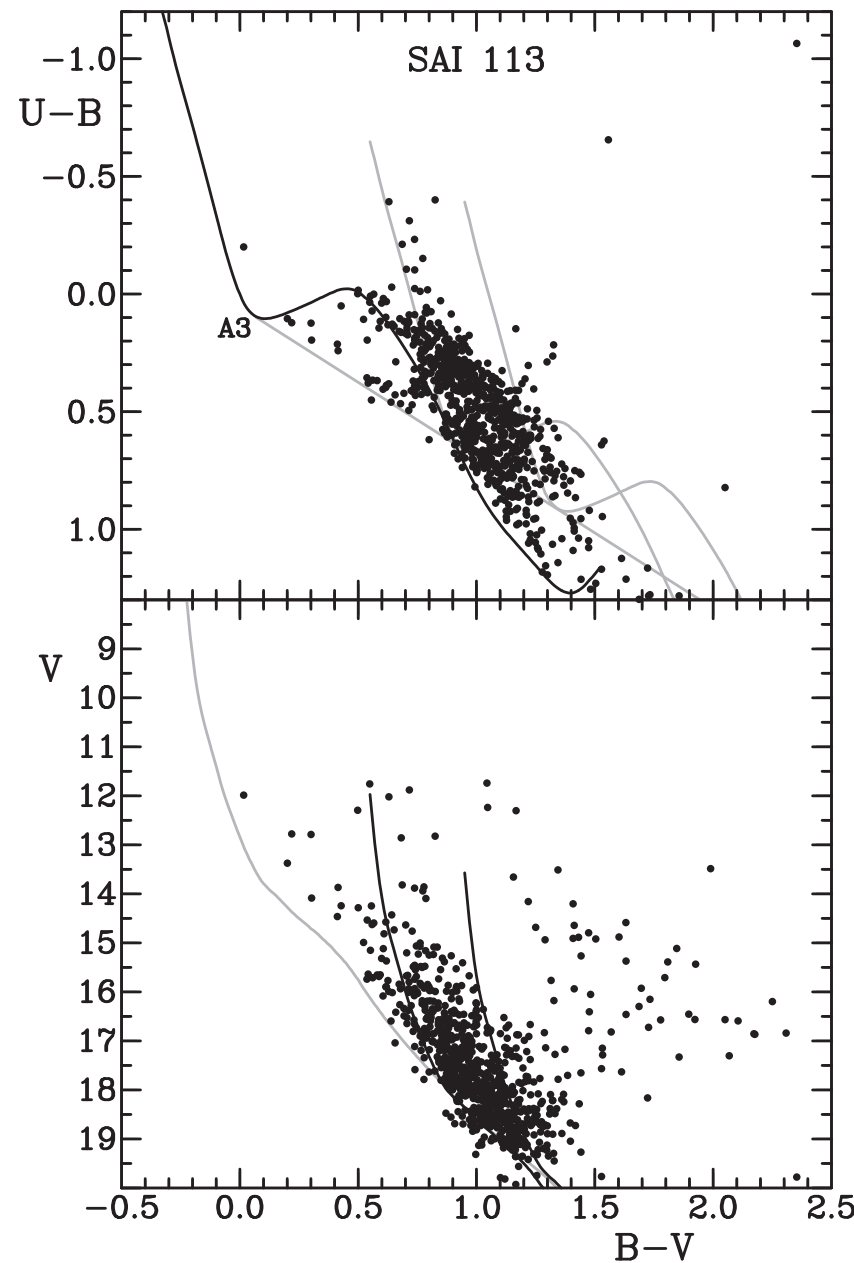

Figure 6. $U B V$ color-color (top) and color-magnitude (bottom) diagrams for stars within $6^{\prime}$ of the center of SAI 113. The black curve (upper) is the intrinsic relation for unreddened stars, while gray curves display its location for reddenings of $E_{B-V}=0.88$ (left) and 1.28 (right). The straight line from the point denoted "A3" is a reddening line of slope $E_{U-B} / E_{B-V}=0.64$. The black curves in the lower diagram are ZAMS isochrones for the previous reddenings and $V_{0}-M_{V}=12.95$. The gray curve in the lower diagram is the ZAMS for $E_{B-V}=0.10$ and an intrinsic distance modulus of $V_{0}-M_{V}=11.83$.

intrinsic $(B-V)_{0}$ colors and reddenings $E_{B-V}$, and corresponding apparent distance moduli $V-M_{V}$ from adoption of ZAMS absolute magnitudes for each star. All color excesses were adjusted to their equivalents for a B0 star using the

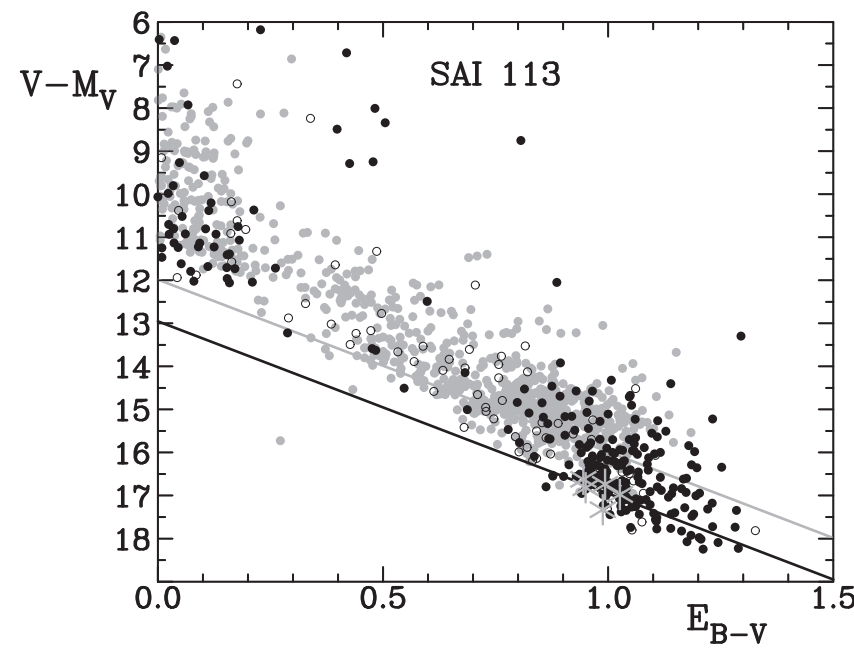

Figure 7. Variable-extinction diagram for SAI 113 tied to ZAMS values of $M_{V}$ for each star (filled circles) and spectroscopic distance moduli (gray asterisks). Similar values are plotted for stars associated with van Genderen 1 (open circles) and stars across the general field (gray points). The black relation of slope $R_{V}=E_{B-V} / A_{V}=4$ and $V_{0}-M_{V}=12.95$ closely fits true ZAMS members of SAI 113, and the gray relation of identical slope with $V_{0^{-}}$ $M_{V}=11.99$ fits ZAMS members in van Genderen 1 and the larger field.

relationship of Fernie (1963). Where ambiguous dereddening solutions were evident, the likely "best" solution was guided by the 2MASS photometry for the star. Any ambiguities that remained were tested using alternate solutions, and in extreme cases were resolved by ignoring the affected star. Although some residual systematics may remain, they are considered minor. Results were isolated according to location in the field: main part of SAI 113, van Genderen 1, and the remainder of the field lying outside the two main groupings. The combined data are shown in the variable-extinction diagram of the field plotted in Figure 7.

The value of $R_{V}$ for the field was established by identifying lower envelopes to the data for both stellar groups, with the dispersion in apparent distance moduli $V-M_{V}$ for each group gauged by eye to be less than \pm 0 . 5 . For SAI 113 this corresponds to the lower section of filled circles in Figure 7, while for van Genderen 1 it was necessary to identify a more luminous lower envelope to the open circles and gray filled circles. Both least squares and non-parametric techniques were applied to the 67 stars identified with the lower envelope of data for SAI 113, in order to reduce potential bias. Least squares methodology generally assumes no uncertainties in one coordinate, while non-parametric techniques make no assumptions about uncertainties in either coordinate. Averaging the results of both techniques weighted according to their resulting uncertainties yielded values of $R_{V}=4.19 \pm 0.22$ and $V_{0}-M_{V}=12.95 \pm 0.11$ (for $R_{V}=4.0 \pm 0.1$ ) for SAI 113, corresponding to a distance of $3.90 \pm 0.19 \mathrm{kpc}$. The data for 221 stars identified with the higher-lying lower envelope of data for van Genderen 1 and the larger field yielded values of $R_{V}=4.06 \pm 0.05$ and $V_{0}-M_{V}=11.98 \pm 0.08$ (for $R_{V}=$ $4.0 \pm 0.1$ ) by the same methodology, corresponding to a distance of $2.49 \pm 0.09 \mathrm{kpc}$.

The color difference method was also applied to the stars in Table 3. Consistent estimates were obtained for stars 39, 187, and 193, yielding a value of $R_{V}=4.22 \pm 0.08$. The 08 dwarf, star 34, and Be star, 85 yielded larger values of $R_{V}$ near 5 and 6, 
Table 4

$R_{V}$ Estimates for Carina

\begin{tabular}{lcl}
\hline \hline Method & \multicolumn{1}{c}{$R_{V}$} & Source \\
\hline SAI 113 VE & $4.19 \pm 0.22$ & This paper \\
van Gend 1 VE & $4.06 \pm 0.05$ & This paper \\
SAI 113 Col. diffs. & $4.22 \pm 0.08$ & This paper \\
Ruprecht 91 VE & $3.82 \pm 0.13$ & Turner et al. (2005) \\
Shorlin 1 CMDs & $4.03 \pm 0.08$ & Turner (2012a) \\
IC 2581 VE & $3.77 \pm 0.19$ & Carraro et al. (2013) \\
West 2 VE & $3.88 \pm 0.18$ & Carraro et al. (2013) \\
West 2 Col. diffs & $3.85 \pm 0.07$ & Carraro et al. (2013) \\
West 2 Spec. fits & $3.77 \pm 0.09$ & Vargas Álvarez et al. (2013) \\
Weighted Mean & $3.99 \pm 0.03$ & $\ldots$ \\
\hline
\end{tabular}

Note. VE = via variable-extinction analysis

which were considered to be affected by emission from circumstellar dust. A value of $R_{V}=4.0 \pm 0.1$ was adopted for the entire region based upon the results of various estimates for $R$ derived here and in other studies of stellar groups in the Carina field (see Table 4).

Many of the same conclusions can be reached from comparable 2MASS color-color and color-magnitude diagrams for stars in SAI 113 plotted in Figure 8, where the intrinsic relations are from Turner (2011), converted from their equivalent reddenings in the $U B V$ system. The addition of an unreddened ZAMS relation corresponding to a distance of 10 $\mathrm{pc}$ is used to indicate that a sizable component of the colormagnitude diagram of Figure 8 originates from unreddened late-type stars lying at distances ranging from 10 to $1000 \mathrm{pc}$ along the line of sight to Carina. Otherwise, the conclusions reached from the $J H K_{s}$ data of Figure 8 match those reached from the $U B V$ data of Figure 6, although with some loss of clarity because of the larger photometric uncertainties, primarily in $H-K$.

A feature of Figure 8 that matches that in Figure 6 is the good fit of the least reddened cluster stars to the ZAMS. But the clump of more highly reddened cluster stars in Figure 8 differs from that in Figure 6, in that it contains many more stars redder than the ZAMS. The reason for this is not clear. This possibly arises from systematic offsets in the 2MASS colors, or may be related to the circumstellar reddening effect that appears in reddened B-type stars in Figure 6. It can be noted that the nuclear radius of 1.5 for SAI 113, noted in Section 1, corresponds to only $1.7 \mathrm{pc}$ at the distance of the cluster, somewhat smaller than values of 2-3 pc, typical of most open clusters. SAI 113 is therefore likely to be a post core-collapse cluster, in other words highly evolved dynamically, consistent with the possible existence of circumstellar reddening for late B-type stars and the generally large main-sequence scatter in the cluster's unreddened color-magnitude diagram (Figure 11).

Similar plots of $U B V$ color-color and color-magnitude diagrams are presented in Figure 9 for the core region of van Genderen 1, and Figure 10 for brighter stars in the general field of Figure 1-namely, the surroundings of van Genderen 1 and SAI 113. Symbols in the two figures are identical to those used in Figure 7, in order to avoid ambiguities. Main-sequence members of van Genderen 1 are clearly less distant than those of SAI 113.

As noted earlier, the least reddened stars in the variableextinction diagram of Figure 7 have intrinsic distance moduli of $V_{0}-M_{V}=11.83$, or $2.3 \mathrm{kpc}$. Yet there is a crude upper envelope

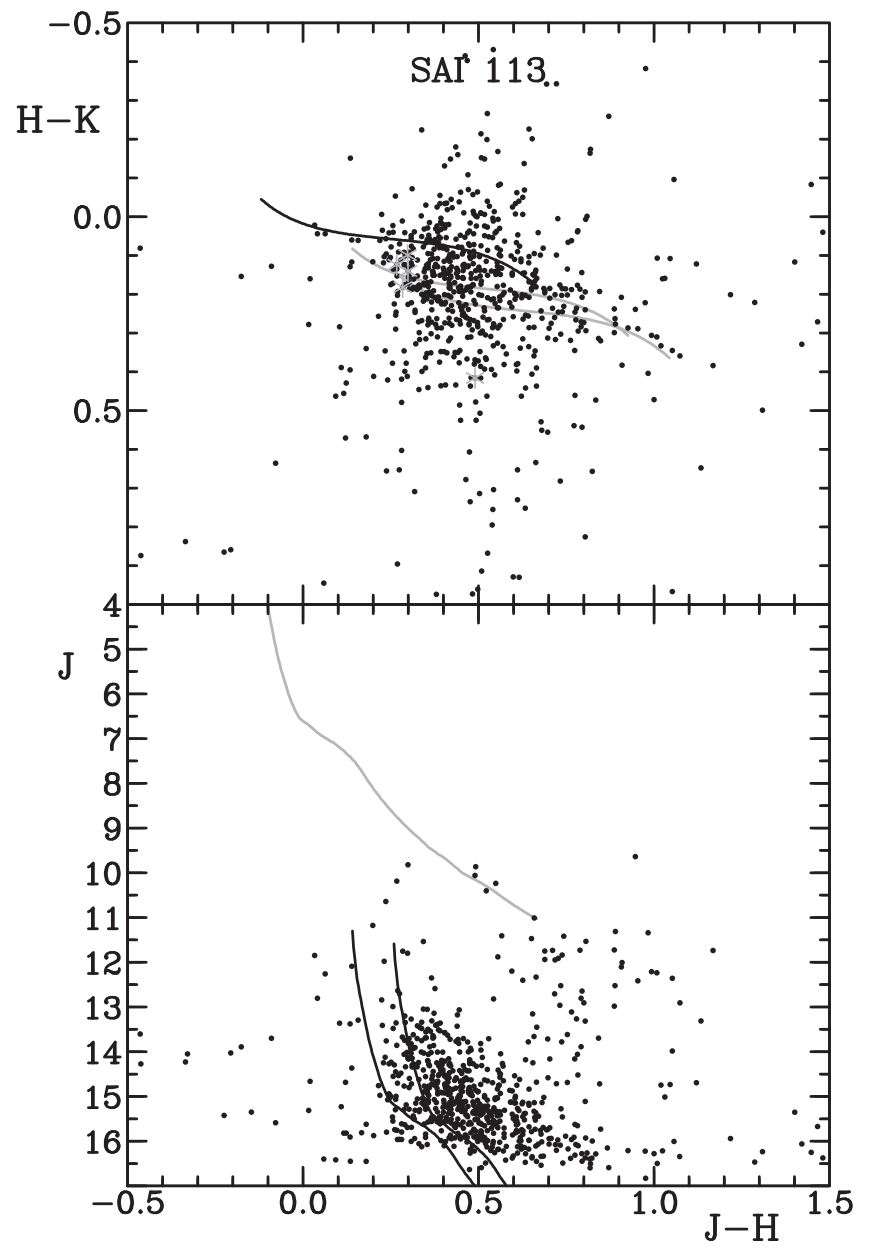

Figure 8. $2 \mathrm{MASS} J H K_{s}$ color-color (top) and color-magnitude (bottom) diagrams for stars in SAI 113, with gray asterisks in the upper portion indicating spectroscopically observed stars. As in Figure 6, the intrinsic relation for unreddened stars is shown by the black curve (upper), and gray curves display its location for reddenings of $E_{B-V}=0.88$ and 1.28. The black curves in the lower diagram are the ZAMS for the previous reddenings and $V_{0}-M_{V}=12.95$. The gray relation is the unreddened ZAMS for a distance of $10 \mathrm{pc}$.

to the ZAMS-fitted stars in Figure 7 near $V_{0}-M_{V} \simeq 10$, or $1 \mathrm{kpc}$, which is where the primary dust extinction begins, according to Neckel \& Klare (1980). Such complications are typical of variable-extinction analyses, and generally arise because of ambiguous dereddening solutions for some stars. The overall conclusions reached from Figures 6-10 are that differential reddening in SAI 113 is relatively small, amounting to only 0.40 in $E_{B-V}$, but that the foreground reddening in SAI 113 is larger than that in van Genderen 1 or across much of the field. Such conclusions match those gleaned from a visual inspection of the field (Figure 3).

An extinction-free color-magnitude diagram is presented for likely members of SAI 113 in Figure 11. Members were selected using the results of the variable-extinction analysis (Figure 7) and spectral classification (Table 3). Post-mainsequence and pre-main-sequence stellar evolutionary model isochrones were interpolated from the results of Meynet et al. (1993) and Palla \& Stahler (1993), as in Turner (2012b). The best match for the upper and lower ends of the main sequence was for $\log t=6.75$, corresponding to an age of $5.6 \times 10^{6}$ years. The strongest restriction on that result is the presence of the $\mathrm{O} 7$ and $\mathrm{O} 8$ dwarfs (stars 34 and 39) in the 


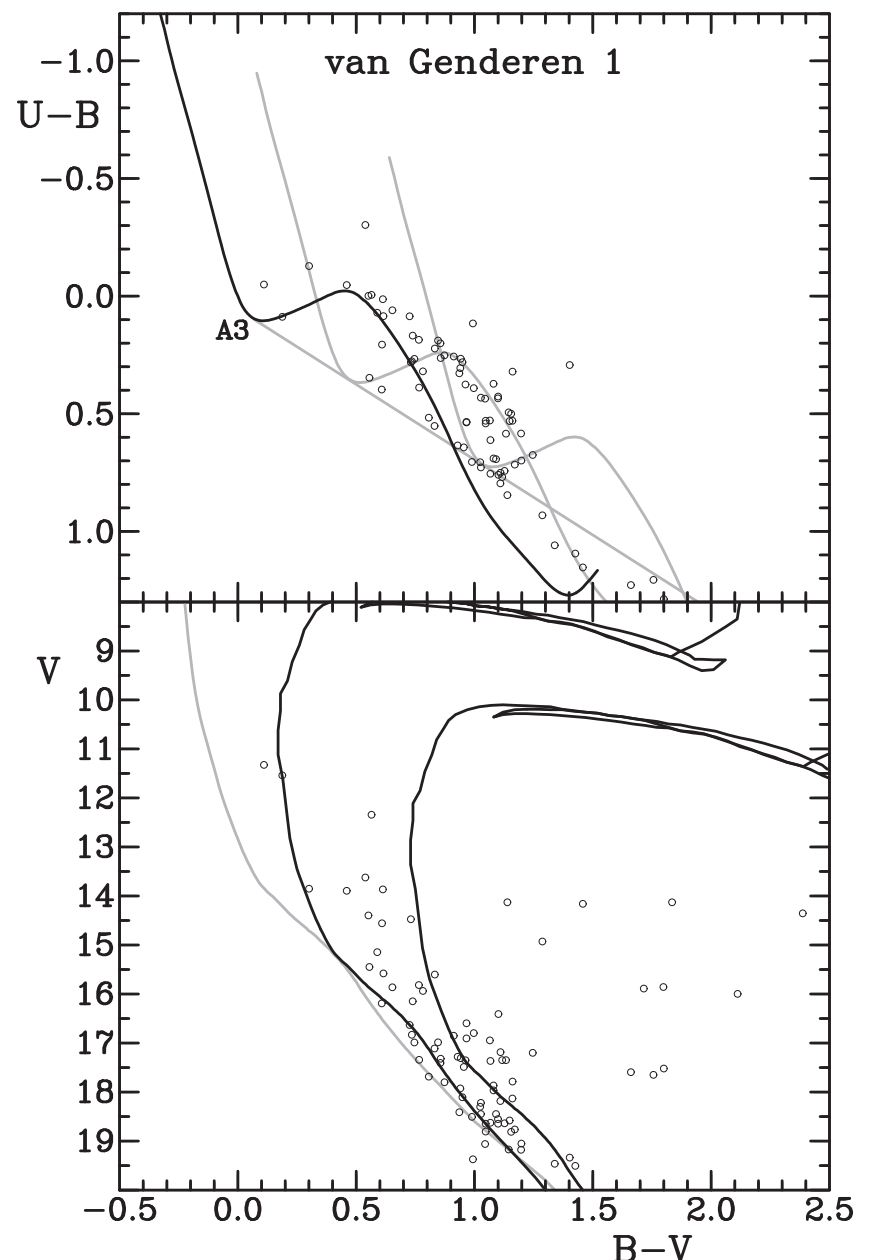

Figure 9. $U B V$ color-color (top) and color-magnitude (bottom) diagrams for stars in the core region of van Genderen 1. Relations are as in Figure 6, except gray curves in the top portion display the intrinsic relation for reddenings of $E_{B-V}=0.41$ (left) and 0.97 (right). The black curves in the lower diagram are $\log t=7.1$ isochrones for the previous reddenings and $V_{0}-M_{V}=11.98$.

cluster. The solid identification of faint cluster members is restricted by the fact that both foreground and background stars projected against SAI 113 share similar reddenings. Stars lying close to or slightly more luminous than the ZAMS in Figure 11 are highly likely members. The advanced dynamical state of SAI 113 explains the large main-sequence scatter as the result of unresolved binaries or, more likely, rapidly rotating stars, possibly from close binary mergers (see Turner 1996).

An extinction-free color-magnitude diagram for likely outlying members of the group of stars associated with van Genderen 1 is presented in Figure 12. The stars lie all across the field of Figure 1, and were selected based upon reddening and where they fell in the variable-extinction diagram (Figure 7). Once again, post-main-sequence and premain-sequence stellar evolutionary model isochrones were taken from those published by Meynet et al. (1993) and Palla \& Stahler (1993). The best match for the upper and lower ends of the main sequence was for $\log t=7.1$, corresponding to an age of $1.3 \times 10^{7}$ years.

Data were included in Figure 12 for the two M-supergiant variables CK Car and EV Car, as summarized in Table 5. The mean light data for the stars were taken from the Hipparcos catalog (ESA 1997), the spectral types are from the General catalog of Variable Stars (Samus et al. 2012), and intrinsic

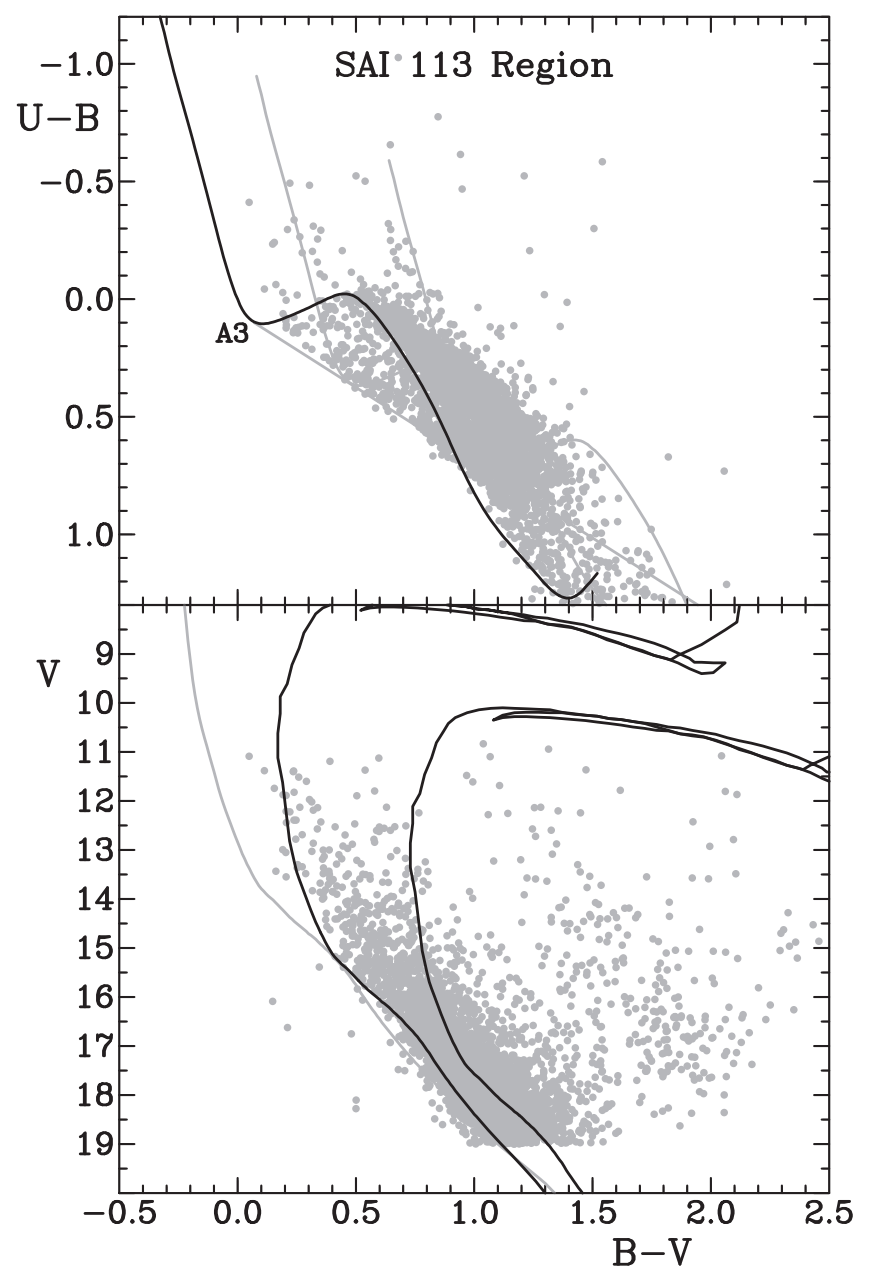

Figure 10. $U B V$ color-color (top) and color-magnitude (bottom) diagrams for stars in the general region surrounding van Genderen 1 and SAI 113. Relations are as in Figure 9.

colors are those of Levesque et al. (2005). The location of the two stars in the color-magnitude diagram of Figure 12 agrees well with the $\log t=7.1$ isochrone, strengthening the case that they may be outlying members of van Genderen 1. Both stars are being investigated for periodicity by D. G. Turner and E. Los using the Harvard College Observatory Photographic Plate Collection, with preliminary results indicating pulsation periods of 518 days for CK Car and 415 days for EV Car. If their outlying membership in van Genderen 1 is confirmed, they become valuable calibrators for the period-luminosity relation for SRC variables (type $\mathrm{C}$ semi-regulars).

\section{Implications for Galactic Structure}

Although the derived parameters for SAI 113 and van Genderen 1 are of interest, particularly with regard to their dynamical histories and stellar membership, of greater importance is how the discovery of a common value of $R_{V}=4$ throughout the Carina region (Table 4) affects the delineation of Galactic spiral arm structure in the fourth quadrant.

Another concern is that the dust lanes running through Carina continue into the adjacent constellations, westward into Vela and Puppis, and eastward into Centaurus and Crux. That is the impression gained from visual inspection of blue light images of the Carina region and its environs. The results of 


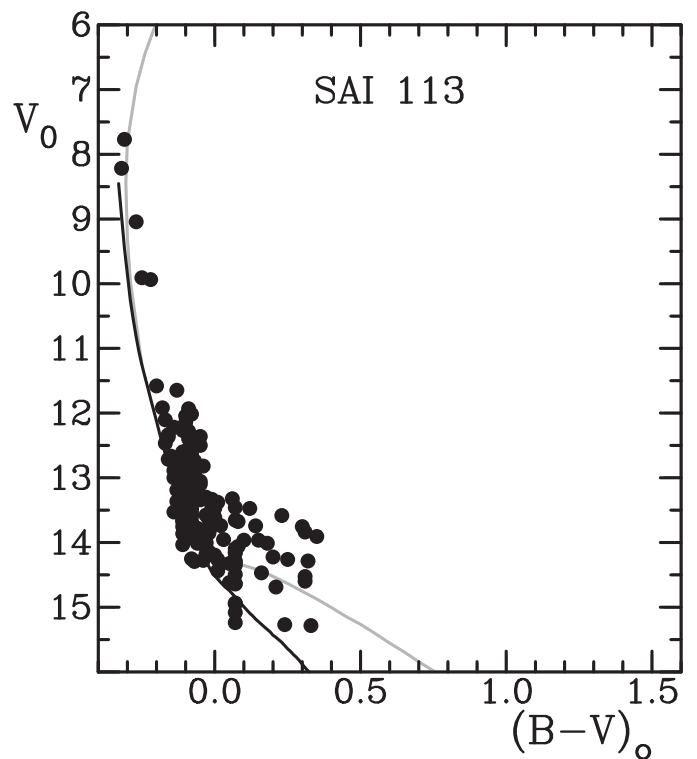

Figure 11. Unreddened color-magnitude diagram for likely members of SAI 113 for an intrinsic distance modulus of $V_{0}-M_{V}=12.95$. The ZAMS is plotted as a black curve, and an isochrone for $\log t=6.75$ as a gray curve.

Neckel \& Klare (1980) suggest that the dust extinction in those regions increases noticeably at distances of about $1 \mathrm{kpc}$ from the Sun, roughly midway between the Sun and the young star complexes in the same fields that help to delineate the Carina spiral arm. A similar impression is given by the more recent three-dimensional dust map of the Galaxy published by Green et al. (2015). Although the Green et al. (2015) map does not include the fourth Galactic quadrant, high dust densities $\sim 1 \mathrm{kpc}$ from the Sun along the borders of the adjoining first and third quadrants appear to be consistent with the Neckel \& Klare (1980) map of nearby extinction, with a dust complex running diagonally across the region $\sim 1 \mathrm{kpc}$ from the Sun. In such a situation one might expect $R_{V} \simeq 4$ throughout much of the fourth Galactic quadrant, at least for regions lying close to the Galactic equator where the dust clouds are concentrated visually.

It is of interest to note that the question of the appropriate value of $R_{V}$ in regions adjacent to Carina was addressed previously, at least for the Centaurus cluster Stock 16, by the study of Vázquez et al. (2005). They argue that $R_{V} \simeq 4$, at least in the regions surrounding the main cluster. That analysis, however, assumed a dependence of the color excess ratio $E_{V-I} / E_{B-V}$ on $R_{V}$ that is incorrect. As demonstrated by Turner et al. (2011b), the ratio $E_{V-I} / E_{B-V}$ is constant (value 1.26) for any value of $E_{U-B} / E_{B-V}$, the latter of which appears to be related to $R_{V}$ (Turner et al. 2014). It is nevertheless possible to demonstrate that $R_{V}=4$ in Stock 16, and that the same value applies in the Galactic plane over a large range of Galactic longitude.

The Stock 16 case can be addressed in the same manner as our study of SAI 113 , by means of $U B V$ and $J H K_{s}$ photometry. Figure 13 presents $U B V$ data for Stock 16 stars, using photoelectric data from Turner (1985) and CCD $B V$ data from the APASS survey of the American Association of Variable Star Observers. A reddening law of slope $E_{U-B} / E_{B-V}=0.64$ was adopted, consistent with the reddening of A3 dwarfs and the $R_{V}$ findings of Vázquez et al. (2005), when combined with the results of Turner et al. (2014). The best reddening for the B stars is $E_{B-V}=0.46$ (top portion of figure), and the best

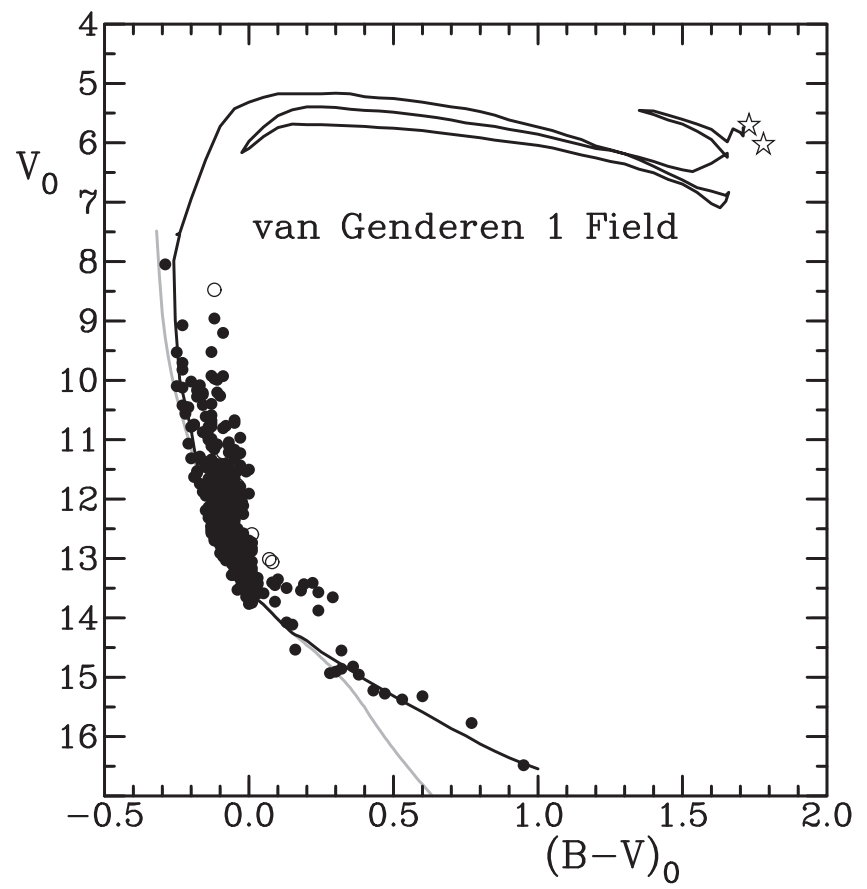

Figure 12. Unreddened color-magnitude diagram for likely members of van Genderen 1 lying in the field of SAI 113 for an intrinsic distance modulus of $V_{0}-M_{V}=11.98$. The ZAMS is plotted as a gray curve, an isochrone for $\log t=7.1$ as a black curve (i.e., the inverse of Figure 11). Open circles represent foreground stars near the main section of SAI 113 that appear to lie foreground to the cluster, and star symbols represent the two M-supergiant variables lying south of van Genderen 1.

ZAMS fit is for $V-M_{V}=12.56 \pm 0.05$. A similar result is obtained with the $U B V$ data of Vázquez et al. (2005).

$J H K_{s}$ data for Stock 16 are shown in Figure 14. A reddening of $E_{B-V}=0.46$ corresponds to $E_{J-H}=0.136$, which is a reasonably good fit to the color-color data for reddened B stars in the top portion of Figure 14. Large uncertainties in the 2MASS colors make it difficult to be more specific. The colormagnitude diagram for Stock 16 in the lower portion of Figure 14 can be interpreted more readily. The best ZAMS fit here is for $J-M_{J}=11.05 \pm 0.05$. The corresponding intrinsic distance moduli, $V_{0}-M_{V}$ (for $R_{V}=4$ ) and $J_{0}-M_{J}$, are both $10.72 \pm 0.05$, corresponding to a distance of $1.393 \pm$ $0.032 \mathrm{kpc}$. Similar results can be obtained using the Vázquez et al. (2005) observations.

Confirmation for the $R_{V}$ value is marginally possible using the variable-extinction method applied to the $U B V$ data for ZAMS stars in Stock 16. The range of color excesses is small, $\sim 0.12$ or slightly larger in $E_{B-V}$, but the methodology of Figure 7 is still suitable. A similar analysis to that of Figure 7 yielded a value of $R_{V}=4.04 \pm 0.49$ for Stock 16, matching the result obtained from ZAMS fitting in $J H K_{s}$ and $U B V$.

Although 2MASS observations are much less precise than typical photoelectric or CCD $U B V$ photometry, they remain extremely useful for deriving reddenings and distances for open clusters (Turner 2011; Turner et al. 2011a; Majaess et al. 2008, 2011, 2012). Infrared photometry also enjoys the advantage of being less susceptible to variations in the extinction law (e.g., color excess ratios and $R_{V}$ ) that are so important for the analysis of optical photometry.

As a test of the conclusions reached here, new distances were derived for young open clusters, mostly in the fourth Galactic quadrant, as a means of determining if the traditional picture of 
Table 5

Data for SRC Variables near van Genderen 1

\begin{tabular}{lccccccc}
\hline \hline Star & $\langle V\rangle$ & $\langle B-V\rangle$ & Sp.T. & $E_{B-V}$ & $(B-V)_{0}$ & $V_{0}$ \\
\hline CK Car & 7.87 & 2.19 & M3.5 Iab & 0.54 & 1.73 & 5.71 \\
EV Car & 7.87 & 2.17 & M4.5 Iab & 0.46 & 1.78 & 6.03 \\
\hline
\end{tabular}

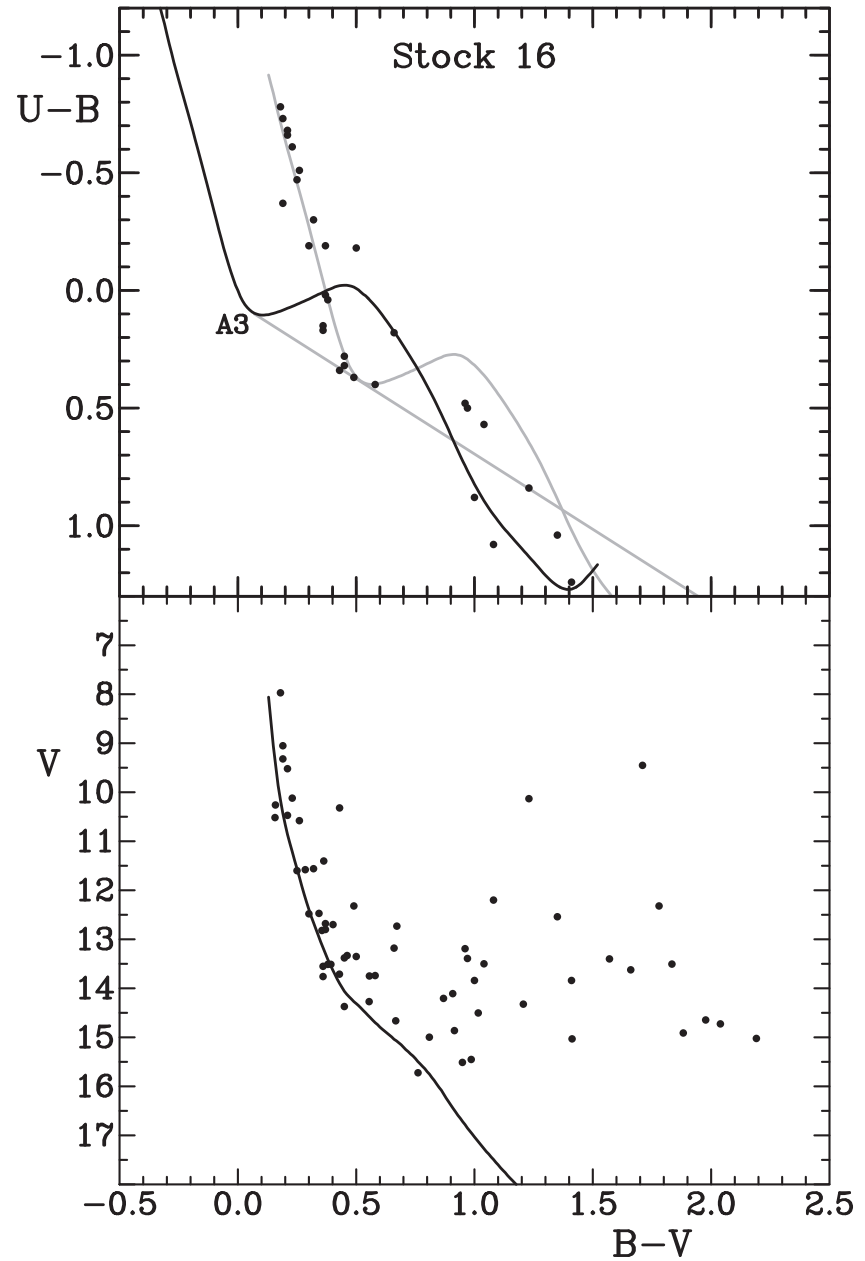

Figure 13. $U B V$ color-color and color-magnitude diagrams for Stock 16, with relations as in Figure 6. The intrinsic relations in both cases are for a reddening of $E_{B-V}=0.46$ and apparent distance modulus of $V-M_{V}=12.56 \pm 0.05$. The implied intrinsic distance modulus for $R_{V}=4$ is $V_{0}-M_{V}=10.72 \pm 0.05$.

spiral arm structure in that quadrant is affected. They were added to similar estimates summarized in Turner (2012a). As in that study, the distances were mainly tied to 2MASS $J H K_{s}$ photometry, but also including existing $U B V$ photometry in the literature, and where necessary, $\mathrm{CCD} B V$ observations from the APASS survey. The combined results are summarized in Table 6, and include a few clusters in the Puppis region. The 2MASS and APASS data for the cluster Hogg 7 were not deep enough to establish a unique solution, mainly because of large differential reddening in the field. The distance to the cluster was therefore left unchanged.

The results are depicted in Figure 15, which displays the spatial location in the Galactic plane of Table 6 clusters, along with a schematic to illustrate the trends indicative of spiral arm features. In this picture, the Carina arm does not run along the line of sight to the Carina clusters, but rather crosses it in nearlinear fashion into Vela. Presumably the Carina arm connects

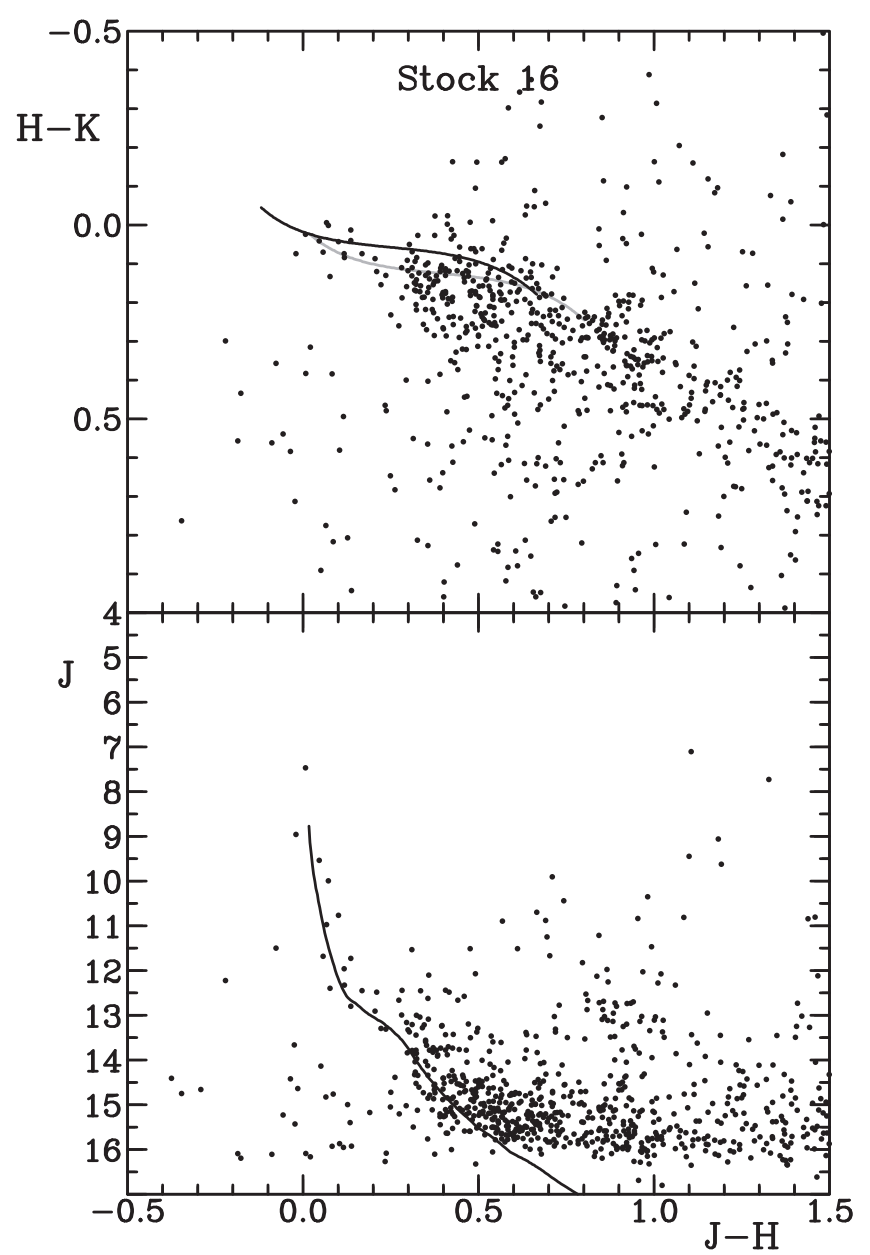

Figure 14. $J H K_{S}$ color-color and color-magnitude diagrams for Stock 16, with relations as in Figure 8. The intrinsic relations in both cases are for a reddening of $E_{J-H}=0.136\left(E_{B-V}=0.46\right)$ and apparent distance modulus of $J-M_{J}=11.05 \pm 0.05$, which corresponds to $J_{0}-M_{J}=10.72 \pm 0.05$, identical to the $U B V$ results in Figure 13.

to the Sagittarius arm, as generally assumed, although in Figure 15 there also appears to be a spur running from it inside the Carina arm. With the older distances to young clusters in the fourth quadrant, the picture of spiral arm structure is much different and less clear.

It may not be possible to confirm the picture of spiral arm structure in the fourth quadrant from $21 \mathrm{~cm} \mathrm{H} \mathrm{I} \mathrm{data,} \mathrm{given} \mathrm{that}$ previous studies have used erroneous corrections of the $\mathrm{HI}$ radial velocities to the Local Standard of Rest (Turner 2014). Additional tests are needed, however.

\section{Summary}

The present study was initiated in order to provide updated parameters for the young open cluster SAI 113 (Skiff 8) in Carina; such parameters having been left unresolved in the Glushkova et al. (2010) study. The field of the CCD $U B V I_{C}$ observations included the nearby group van Genderen 1, which 
Table 6

New Distances to Southern Young Open Clusters

\begin{tabular}{|c|c|c|c|c|}
\hline Cluster & $\ell\left(^{\circ}\right)$ & $b\left(^{\circ}\right)$ & $d_{\mathrm{Lit}}(\mathrm{kpc})$ & $d_{\text {new }}(\mathrm{kpc})$ \\
\hline NGC 6611 & 16.9540 & 0.7934 & 2.04 & . \\
\hline Waterloo 3 & 242.562 & 1.4434 & 5.20 & 2.63 \\
\hline AQ Pup & 246.1562 & 0.1061 & 3.21 & $\ldots$ \\
\hline Pismis 11 & 271.6567 & -0.7077 & 3.60 & 2.46 \\
\hline Ruprecht 79 & 277.0986 & -0.8180 & 1.98 & 3.09 \\
\hline NGC 3105 & 279.9147 & 0.2636 & 8.53 & 2.70 \\
\hline ASCC 58 & 281.7116 & 1.3281 & 0.60 & 0.67 \\
\hline Loden 27 & 282.1710 & -0.3346 & 2.63 & 2.20 \\
\hline Loden 28 & 282.2139 & -2.2145 & 3.95 & 2.34 \\
\hline IC 2581 & 284.5880 & 0.0350 & 2.87 & 2.08 \\
\hline Loden 112 & 284.6870 & 1.1641 & 2.50 & 2.13 \\
\hline SAI 113 & 285.0554 & -1.8883 & $\ldots$ & 3.90 \\
\hline van Genderen 1 & 285.1145 & -2.0270 & $\ldots$ & 2.49 \\
\hline Ruprecht 90 & 285.4175 & -0.4372 & 6.00 & 5.14 \\
\hline NGC 3293 & 285.8527 & 0.0717 & 2.50 & 2.25 \\
\hline NGC 3324 & 286.2283 & -0.1884 & 3.12 & 2.08 \\
\hline Hogg 7 & 286.3948 & -2.5335 & 4.58 & $\mathrm{NC}$ \\
\hline Trumpler 14 & 287.4081 & -0.5774 & 3.10 & 2.17 \\
\hline Trumpler 15 & 287.4100 & -0.3688 & 2.60 & 2.06 \\
\hline Collinder 232 & 287.4929 & -0.5436 & 3.00 & 0.99 \\
\hline Trumpler 16 & 287.6238 & -0.6517 & 3.39 & 2.06 \\
\hline Collinder 228 & 287.6677 & -1.0471 & 2.51 & 1.95 \\
\hline Pismis 17 & 289.4693 & 0.1394 & 3.50 & 1.87 \\
\hline Sher 1 & 289.6373 & -0.2419 & 5.88 & 3.74 \\
\hline Feinstein 1 & 290.0251 & 0.3847 & 1.16 & 2.10 \\
\hline Loden 306 & 290.5547 & -0.7845 & 2.00 & 1.83 \\
\hline Shorlin 1 & 290.6314 & -0.9072 & $\ldots$ & 2.94 \\
\hline NGC 3572 & 290.7047 & 0.2022 & 2.00 & 1.83 \\
\hline NGC 3766 & 294.1169 & -0.0304 & 2.21 & 1.77 \\
\hline NGC 4103 & 297.5736 & 1.1551 & 1.63 & 1.52 \\
\hline Hogg 15 & 302.0474 & -0.2417 & 3.20 & 1.96 \\
\hline NGC 4755 & 303.2057 & 2.5089 & 1.98 & 1.80 \\
\hline Stock 16 & 306.1486 & 0.0631 & 1.90 & 1.39 \\
\hline Hogg 16 & 307.4772 & 1.3372 & 1.59 & 1.75 \\
\hline Collinder 272 & 307.5947 & 1.2016 & 2.10 & 1.79 \\
\hline NGC 6383 & 355.6897 & 0.0412 & 0.99 & 0.96 \\
\hline
\end{tabular}

Note. $\mathrm{NC}=$ no change from literature value.

was examined as well. Both young groups are relatively young, with ages of $5.6 \times 10^{6}$ years and $1.3 \times 10^{7}$ years, respectively, lying at distances of $3.90 \pm 0.19 \mathrm{kpc}$ and $2.49 \pm 0.09 \mathrm{kpc}$. SAI 113 displays evidence for substantial dynamical evolution, and appears to have begun dissolution into the general field. The group designated as van Genderen 1 appears to comprise the remains of a former star cluster that has already lost most of its members, which are spread across the surrounding field and nearby SAI 113. Two potential outlying members of van Genderen 1 are the M-supergiant variables CK Car and EV Car, which could become valuable calibrators for the SRC period-luminosity relation.

An important consequence of the photometric study of the cluster fields is the confirmation that the extinction law is described by a reddening relation of slope $E_{U-B} / E_{B-V}=0.64$ and a ratio of total-to-selective extinction of $R_{V}=4$, identical to what has been found for other young clusters in Carina. Moreover, such "anomalous" extinction appears to extend to adjacent regions of the Galactic plane, affecting how we view the spiral arm structure in the fourth Galactic quadrant. It appears that the Carina spiral feature does not extend along the Galactic line of sight into Carina, but crosses it in roughly

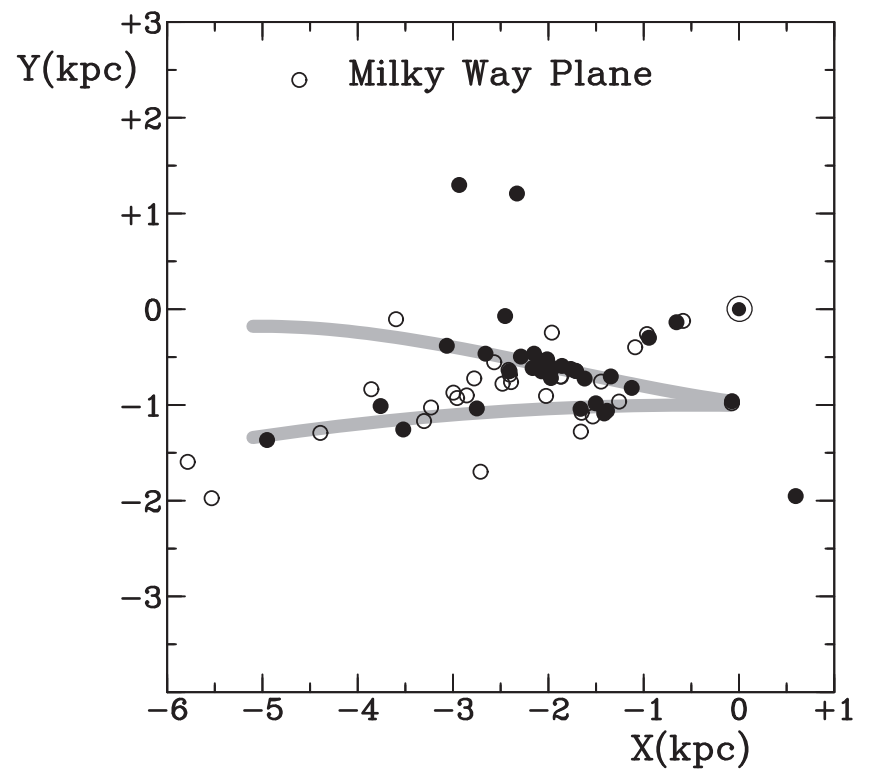

Figure 15. Plot of Galactic plane projection for the young clusters summarized in Table 6 , with new distances denoted by filled circles, literature distances by open circles, and a Sun-symbol indicating the location of the Sun. Gray curves are used to suggest how young open clusters may be connected to map spiral arms in the fourth quadrant.

linear fashion, continuing into Vela. The arm is only about $2 \mathrm{kpc}$ distant in this direction, rather than $2.5-3.0 \mathrm{kpc}$, as is sometimes believed (see Davidson \& Humphreys 1997), with the extinction apparently originating at distances of about $1 \mathrm{kpc}$.

Clearly, how we interpret extinction in various segments of the Galactic plane directly affects the accurate derivation of distances to stellar groups such as the young open clusters normally employed as spiral arm tracers. That point was first made by Johnson (1968) a half century ago in connection with the Galaxy's Perseus arm, but is still appropriate today, at least with regard to how spiral features are mapped in the fourth Galactic quadrant.

This publication makes use of data products from the Two Micron All Sky Survey, which is a joint project of the University of Massachusetts and the Infrared Processing and Analysis Center/California Institute of Technology, funded by the National Aeronautics and Space Administration and the National Science Foundation. The study is also based partly on observations obtained at the Gemini South Observatory (program GS-2016A-Q-109, P.I. Alejo), which is operated by the Association of Universities for Research in Astronomy, Inc., under a cooperative agreement with the NSF on behalf of the Gemini partnership: the National Science Foundation (United States), the National Research Council (Canada), CONICYT (Chile), Ministerio de Ciencia, Tecnología e Innovación Productíva (Argentina), and Ministério da Ciência, Tecnologia e Inovação (Brazil).

\section{References}

Baume, G., Carraro, G., Comeron, F., \& de Elia, G. C. 2011, A\&A, 531, 73 Baume, G., Carraro, G., \& Momany, Y. 2009, MNRAS, 398, 221 Carraro, G., Turner, D., Majaess, D., et al. 2013, A\&A, 555, A50 
Cutri, R. M., Skrutskie, M. F., van Dyk, S., et al. 2003, NASA/IPAC Infrared Science Archive, The IRSA 2MASS All-Sky Point Source Catalog of Point Sources, http://irsa.ipac.caltech.edu/applications/Gator/

Davidson, K., \& Humphreys, R. M. 1997, ARA\&A, 35, 1

ESA 1997, The Hipparcos and Tycho Catalogues, ESA SP-1200

Fernie, J. D. 1963, AJ, 68, 780

Glushkova, E. V., Koposov, S. E., Zolotukhin, I. Yu., et al. 2010, AstL, 36, 75

Green, G. M., Schlafly, E. F., Finkbeiner, D. P., et al. 2015, ApJ, 810, 25

Henize, K. H. 1976, ApJS, 30, 491

Johnson, H. L. 1968, in Nebulae and Interstellar Matter, ed. y. B. M. Middlehurst \& L. H. Aller (Chicago: Univ. Chicago Press), 167

Kholopov, P. N. 1969, SvA, 12, 625

Landolt, A. U. 1992, AJ, 104, 340

Levesque, E. M., Massey, P., Olsen, K. A. G., et al. 2005, ApJ, 628, 973

Maíz Apellániz, J., Sota, A., Arias, J. I., et al. 2016, ApJS, 224, 1 id. 4

Majaess, D. J., Turner, D. G., Gallo, L., et al. 2012, ApJ, 753, 144

Majaess, D. J., Turner, D. G., \& Lane, D. J. 2008, MNRAS, 390, 1539

Majaess, D. J., Turner, D. G., Lane, D. J., \& Krajci, T. 2011, JAVSO, 39, 219

Meynet, G., Mermilliod, J.-C., \& Maeder, A. 1993, A\&AS, 98, 477

Neckel, Th., \& Klare, G. 1980, A\&AS, 42, 251

Palla, F., \& Stahler, S. W. 1993, ApJ, 418, 414

Samus, N. N., Antipin, S. V., Durlevich, O. V., et al. 2012, General Catalog of Variable Stars database, Version 2012Apr (Institute of Astronomy of Russian Academy of Sciences and Sternberg State Astronomical Institute of the Moscow State University)

Schild, R. F., \& Cowley, A. P. 1971, A\&A, 14, 66

Schild, R. F., \& Romanishin, W. 1976, ApJ, 204, 493

Skrutskie, M. F., Cutri, R. M., Stiening, R., et al. 2006, AJ, 131, 1163

Stetson, P. B. 1987, PASP, 99, 191
Stetson, P. B. 1992, JRASC, 86, 71

Turner, D. G. 1976a, AJ, 81, 97

Turner, D. G. 1976b, AJ, 81, 1125

Turner, D. G. 1979, PASP, 91, 642

Turner, D. G. 1985, ApJ, 292, 148

Turner, D. G. 1989, AJ, 98, 2300

Turner, D. G. 1993, A\&AS, 97, 755

Turner, D. G. 1996, in ASP Conf. Ser. 90, The Origins, Evolution, and Destinies of Binary Stars, ed. E. F. Milone \& J.-C. Mermilliod (San Francisco, CA: ASP), 382

Turner, D. G. 2011, RMxAA, 47, 127

Turner, D. G. 2012a, Ap\&SS, 337, 303

Turner, D. G. 2012b, AN, 333, 174

Turner, D. G. 2014, CaJPh, 92, 959

Turner, D. G. 2016, RMxAA, 52, 223

Turner, D. G., Forbes, D., van den Bergh, S., Younger, P. F., \& Berdnikov, L. N. 2005, AJ, 130, 1194

Turner, D. G., Kovtyukh, V. V., Majaess, D. J., et al. 2009, AN, 330, 807

Turner, D. G., MacLellan, R. F., Henden, A. A., \& Berdnikov, L. N. 2011b, RMxAA, 47, 345

Turner, D. G., Majaess, D. J., \& Balam, D. D. 2014, CaJPh, 92, 1696

Turner, D. G., Rosvick, J. M., Balam, D. D., et al. 2011a, PASP, 123, 1249

van den Bergh, S., \& Sher, D. 1960, PDDO, 2, 7

van Genderen, A. M., Robijn, F. H. A., van Esch, B. P. M., \& Lamers, H. J. G. 1991, A\&A, 246, 407

Vargas Álvarez, C. A., Kobulnicky, H. A., Bradley, D. R., et al. 2013, AJ, 145,125

Vázquez, R. A., Baume, G. L., Feinstein, C., Nuñez, J. A., \& Vergne, M. M. 2005, A\&A, 430, 471 